\title{
Practical method for modelling the nonlinear behaviour of a travelling wave semiconductor optical amplifier
}

\author{
S. Ruiz-Moreno \\ J. Guitart
}

Indexing terms: Optical amplifiers, Semiconductor devices and materials

\begin{abstract}
The authors present a simple model of a travelling wave semiconductor optical amplifier with an appreciable degree of saturation. The model uses a particular way of linearising the total carrier recombination $R(N)$ to find useful expressions for the saturation parameter and/or the carrier lifetime. By combining these expressions and the gain measurements realised, it is possible to establish the dependence between the mentioned parameters and the input optical power.
\end{abstract}

\section{Introduction}

The main goal of this paper is to investigate some aspects related to the nonlinear behaviour of a travelling wave semiconductor optical amplifier (TWOA) (negligible residual reflectivities). Its main characteristics and performances (gain, bandwidth and noise figure) have been analysed by several authors for bulk structures (see, for example, References 1-3), as well as for quantum-well structures $[4,5]$. These latter structures (QW-TWOA) present a narrower spectral gain, a lower noise figure and high saturation parameter, although, on the other hand, conventional optical amplifiers (TWOA) have a more simple fabrication process.

To determine the performances that such devices can offer, it is necessary to develop realistic models to describe the light amplification process. This becomes particularly complicated when the unavoidable nonlinear behaviour of the semiconductor amplifier must be taken into account. In such cases, the concept of saturation parameter $\left(P_{s}\right)$ becomes very useful in establishing the relationship between the input and output optical powers $[3,4]$. Nevertheless, owing to the total recombination mechanism in the active layer (nonradiative recombination, spontaneous emission and Auger recombination), it is difficult to determine the value of this parameter with precision. Actually, it depends on the propagation coordinate and therefore on the input optical power. This is an important aspect that will be considered here (see also Reference 9).

In this paper, we present a practical method of measuring (in an indirect way) the value of the saturation parameter and/or the effective carrier lifetime $\left(\tau_{c}\right)$. It is an

Paper 9325J (E3, E13), first received 7th July and in revised form 17th November 1992

The authors are with the Signal Theory \& Communications Department, Polytechnic University of Catalonia, Modul D5 Campus Nord UPC, c/Sor Eulalia de Anzizu s/n, 08034 Barcelona, Spain

IEE PROCEEDINGS-J, Vol. 140, No. 1, FEBRUARY 1993 indirect method as the magnitude we have actually measured in the laboratory [6] has been the optical power at the amplifier output. Measurements have been done for an appreciable degree of saturation and in a wide range of input power values (from $-26 \mathrm{dBm}$ to $-5 \mathrm{dBm}$ ).

Using the set of the obtained experimental measurements and a certain proposed model for the recombination, we have calculated $P_{s}$ and $\tau_{c}$ as a function of the incident optical power. The developed model (see Section 2) linearises the total carrier recombination around a value that is easily calculable starting from the gain measurements realised

\section{Proposed model for a nonlinear TWOA}

The nonlinear behaviour of a travelling wave semiconductor optical amplifier can be described starting from the rate equation for the local carrier density $N(z, t)$. This equation has the form [7]

$$
\frac{d N(z, t)}{d t}=\frac{I}{q V}-R[N(z, t)]-\frac{\Gamma g_{m}[N(z, t)]}{h f W d} P(z, t)
$$

where $z$ is the amplification co-ordinate; $I$ is the current injected in the active region (volume $V$ ); $q$ is the electron charge; $h f$ is the photon energy; $P(z, t)$ is the optical power travelling through the active region (cross-section $W d$ ); and $\Gamma$ is the confinement factor. The material gain $g_{m}$ (per unit length) can be linearly expressed as a function of the carrier density by

$$
g_{m}[N(z, t)]=a\left[N(z, t)-N_{t}\right]
$$

where $N_{t}$ is the carrier density at the transparency level of the material having a gain coefficient $a$. The total recombination rate can be expressed as [8]

$$
R(N)=A N+B N^{2}+C N^{3}
$$

$A, B$ and $C$ being the nonradiative recombination, the spontaneous emission and the Auger recombination terms, respectively. It must be pointed out that an accurate knowledge of these constants is certainly hard from a technological point of view.

The authors are grateful to the Départment FOG of the CNET Lannion (France), and especially to Dr. J.C. Simon, for allowing them to realise experimental measurements. This work has been supported by RACE Project 1027 WP.4 (Optical Amplifiers) 
In a stationary case (CW input signals), eqn. 1 becomes

$$
\frac{I}{q V}=R[N(z)]+\frac{\Gamma g_{m}[N(z)]}{h f W d} P(z)
$$

Now, our goal is to find an analytical expression for $g_{m}[N(z)]$ as a function of the optical power $P(z)$ as, in this way, it is possible to establish a definition for the saturation parameter. Unfortunately, this is only possible assuming a linear variation between $R$ and $N$, which is strictly true only when $t_{c}$ is a constant. This is equivalent to putting $R=N / \tau_{c}$ in eqn. 4 , and, in such a case, the expression for $P_{s}$ is given by the well-known formula [3]

$$
P_{s}=\frac{h f W d}{\Gamma a \tau_{c}^{0}}
$$

where $\tau_{c}^{0}$ represents a constant carrier lifetime. In addition to the assumption of a linear recombination, note that this expression does not take into account other losses mechanisms such as, for instance, those due to the intervalence band absorption. If such an effect is to be considered (see for example, Reference 10), the denominator of expr. 5 should be replaced by $\Gamma\left(a-K_{0}\right) \tau_{c}^{o}$, where $K_{0}$ is the intervalence band absorption coefficient.

In another work, Jopson and Darcie [7] propose an expansion of $R(N)$ in a Taylor series around an average carrier density name $N_{0}$. This requires the previous knowledge of $N(z)$ by means of a relatively complicated numerical procedure. To find practical expressions for $P_{s}$ or $\tau_{c}$, we also propose to linearise the function $R(N)$ around a value of $N$ but defined as

$$
\bar{N} \equiv \frac{1}{2}\left(N_{0}+N_{L}\right)
$$

$N_{0}$ and $N_{L}$ being the local carrier densities at the input $(z=0)$ and at the output of the amplifier ( $z=L=$ amplifier length), respectively.

According to eqn. 3, we can write the derivative of $R(N)$ as

$$
R^{\prime}(N)=A+2 B N+3 C N^{2}
$$

and, consequently,

$$
R^{\prime}(N)=A+B\left(N_{0}+N_{L}\right)+3 C\left(\frac{N_{0}+N_{L}}{2}\right)^{2}
$$

The approximation we propose for $R(N)$ is

$$
R(N) \approx R(\bar{N})+(N-\bar{N}) R^{\prime}(\bar{N})
$$

Now, we need to determine the values of $N_{0}$ and $N_{L}$ which, in turn, will determine $\bar{N}$ according to eqn. 6 . This is the key point that justifies the advantages of the proposed method. In effect, starting from eqn. 4, we can easily establish the following boundary conditions at $z=0$ and $z=L$

$$
\begin{aligned}
& \frac{I}{q V}=R\left(N_{0}\right)+\frac{\Gamma a\left(N_{0}-N_{t}\right)}{h f W d} P(0) \\
& \frac{I}{q V}=R\left(N_{L}\right)+\frac{\Gamma a\left(N_{L}-N_{t}\right)}{h f W d} P(L)
\end{aligned}
$$

Taking into account expr. 3 , eqns. 10 easily allows the values of $N_{0}$ and $N_{L}$ to be known. In this way, we can determine $\bar{N}$ with eqn. $6, R(\bar{N})$ with eqn. 3 and $R^{\prime}(\bar{N})$ with eqn. 7. It must be emphasised that $P(0)=P_{I N}$ and $P(L)=P_{\text {oUT }}$ can be directly deduced from the experimental $\boldsymbol{P}_{I N}-\boldsymbol{P}_{\text {OUT }}$ characteristic of the amplifier presented in Section 3. Consequently, at this moment, we already know the linearised expression of the total carrier recombination, and therefore we are in a position to find the relationship between $g_{m}$ and $\boldsymbol{P ( z )}$.

Replacing approximation 9 in eqn. 4 , and after some algebraic manipulations have been done, it is simple to obtain the following expression:

$$
g_{m}[P(z)]=\frac{[(I / q V)-R(\bar{N})] / R^{\prime}(\bar{N})+\bar{N}-N_{t}}{1+P(z) /\left[\frac{h f W d}{\Gamma a} R^{\prime}(\bar{N})\right]} a
$$

which shows the dependence of the material gain (per unit length) on the optical power during the amplification process. It is well known that, in the right-hand side of eqn. 11 , the second term in the denominator represents the amplifier saturation, and the numerator is independent of the $z$-co-ordinate and tends to $a\left(\bar{N}-N_{t}\right)$ when $P_{I N}$ tends to zero. Therefore, we can establish that the term

$$
P_{s} \equiv \frac{h f W d}{\Gamma a} R^{\prime}(\bar{N})
$$

is the saturation parameter of the amplifier. Here we note that, if the intervalence band absorption had been considered, the expression for $P_{s}$ would be

$$
P_{\mathrm{s}} \equiv \frac{h f W d}{\Gamma\left(a-K_{0}\right)} R^{\prime}(\bar{N})
$$

Using eqns. 8 and $12 a$, we obtain

$$
P_{s}=\frac{h f W d}{\Gamma a}\left[A+B\left(N_{0}+N_{L}\right)+3 C\left(\frac{N_{0}+N_{L}}{2}\right)^{2}\right]
$$

In this formula, $N_{0}$ and $N_{L}$ can be calculated from eqns. $10 a$ and $10 b$, respectively, for each pair of experimental $P_{I N}-P_{\text {oUT }}$ values.

The effective carrier lifetime can be also calculated in a simple way. For example, according to Reference 7 , we can define

$$
\tau_{c} \equiv \frac{1}{R^{\prime}(\bar{N})}
$$

and therefore

$$
\tau_{c}=\left[A+B\left(N_{0}+N_{L}\right)+3 C\left(\frac{N_{0}+N_{L}}{2}\right)^{2}\right]^{-1}
$$

It is obvious that both $P_{s}$ and $\tau$ depend intrinsically on the value $\bar{N}$ around which $R(N)$ has been expanded. A different option to the one used in this paper is the expansion of $R(N)$ in a Taylor series around

$$
\bar{N} \equiv \frac{1}{L} \int_{0}^{L} N(z) d z
$$

This option presents the drawback that the carrier distribution along the amplifier $N(z)$ must be known a priori, which implies the resulting iterative procedure for convergence. Actually, there are two problems. On the one hand, there is a value of $\tau_{c}$ (and $\boldsymbol{P}_{s}$ ) for each amplifier co-ordinate $(z)$. On the other hand, from a mathematical point of view (see eqn. 4), it is not possible to define $\tau_{c}$ (or $P$ ) without using a linear approximation of $R(N)$ around some value of $N$. With reference to this, we have chosen $\bar{N}=\left(N_{0}+N_{L}\right) / 2$, owing to the practical reasons mentioned above. In any case, as the results do not change significantly according to the definitions given in eqns. 6 or 16 , we believe that the important fact is the dependence of both $\tau_{c}$ and $P_{s}$ on the input optical power. This

IEE PROCEEDINGS-J, Vol. I40, No. I, FEBRUARY 1993 
will be shown in Section 4. In the following Section, we present the experimental characterisation carried out for the TWOA under study.

\section{Experimental input/output characteristic of the}

In this Section, we present the experimental results obtained from the measurements carried out for the TWOA, a double-channel planar buried heterostructure type (DCPBH), grown by a molecular beam epitaxy process (MBE). The sample we have characterised was manufactured by Alcatel Alsthom for RACE Project 1027 WP.4 (Optical Amplifiers), and we made the measurements at CNET laboratories in Lannion (France) [6] The main parameters of this optical amplifier are shown in Table 1. It must be pointed out that, in fact, it is a near

Table 1: Characteristic parameters of the TWOA under study (Sample 3715 \# J23, RACE Project 1027 WP.4)

\begin{tabular}{|c|c|c|}
\hline Symbol & Parameter & Value \\
\hline$A$ & nonradiative recombination & $\begin{array}{l}\text { negligible (at room } \\
\text { temperature) }\end{array}$ \\
\hline$B$ & spontaneous recombination & $1.3 \times 10^{-16} \mathrm{~m}^{3} / \mathrm{s}$ \\
\hline$C$ & Auger recombination & $4 \times 10^{-41} \mathrm{~m}^{6} / \mathrm{s}$ \\
\hline$L$ & amplifier length & $810 \mu \mathrm{m}$ \\
\hline$w$ & active region width & $1.2 \mu \mathrm{m}$ \\
\hline$d$ & active layer thickness & $0.13 \mu \mathrm{m}$ \\
\hline $\bar{r}$ & confinement factor & 0.23 \\
\hline$a$ & gain coefficient & $2.5 \times 10^{-20} \mathrm{~m}^{2}$ \\
\hline$h f$ & photon energy & $1.3 \times 10^{-19} \mathrm{~J}$ \\
\hline 1 & injected current & $112 \mathrm{~mA}$ \\
\hline$N_{t}$ & carrier density at transparency & $1.023 \times 10^{24} / \mathrm{m}^{3}$ \\
\hline
\end{tabular}

travelling wave optical amplifier (NTWOA) treated with an antireflection coating process to achieve residual facet reflectivities close to $10^{-5}$

The signal optical power at the amplifier output is plotted in Fig. 1 as a function of the incident optical

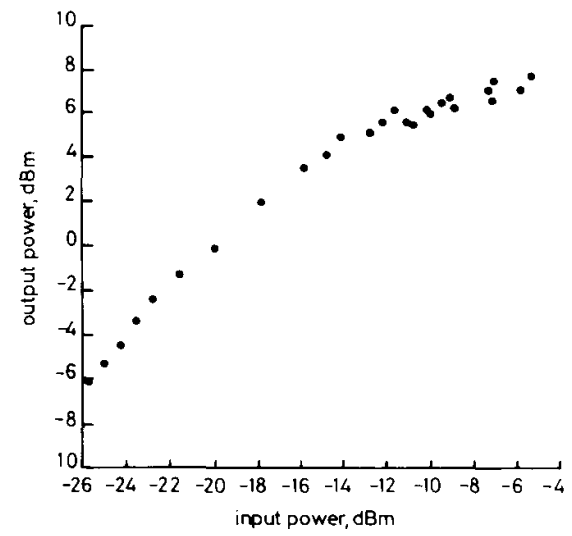

Fig. 1 Amplifier output power $P_{O U T}$, measured experimentally, as a function of the input power $P_{I N}$

power (CW signal) at $1530 \mathrm{~nm}$ (wavelength of the material peak gain). To achieve an appreciable nonlinear behaviour, the measurements have been realised from $P_{I N} \approx-26 \mathrm{dBm}(\approx 2.5 \mu \mathrm{W})$ up to $-5 \mathrm{dBm}(\approx 0.3 \mathrm{~mW})$. All the dots in Fig. 1 correspond to real optical powers at the input and output of the amplifier. This means that the slope of the $P_{I N}-P_{O U T}$ characteristic shown is the actual single pass gain $\left(G_{s}\right)$ of the device. The measurements indicate that, for small values of the input power,

IEE PROCEEDINGS-J, Vol. 140, No. I, FEBRUARY 1993 the amplifier has $G_{s} \approx 20 \mathrm{~dB}$, which is in good agreement with the theoretical value of the small signal gain. Note that the gain decreases by $3 \mathrm{~dB}$ when $P_{I N} \approx-11 \mathrm{dBm}$.

On the other hand, it can be pointed out that the number of measurements realised is greater in the range where the saturation is more important. This is owing to the fact that the laser source we used (high-power laser) showed some instabilities at high power levels, and therefore it became necessary to have available some extra measurements to deduce the actual behaviour of the amplifier with more accuracy.

In the following Section, we will use the experimental results presented here and the model developed in the preceding Section to calculate $\tau_{c}$ and $P_{s}$.

\section{Results and discussion}

Some experimental results shown here were already exposed in Reference 9, although now the optical power range we are going to consider is larger.

First, we have calculated (see Fig. 2) the values of $N_{0}$ $N_{L}$ and $\bar{N}$ as a function of the incident optical power

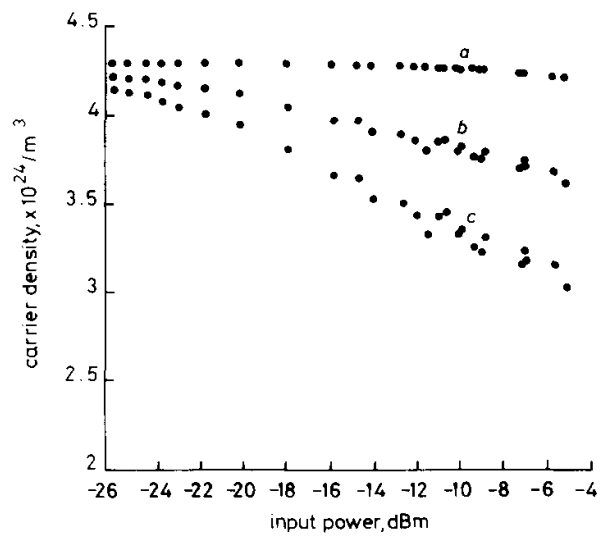

Fig. 2 Local carrier densities $N_{0}, N_{L}$, and averaged carrier density $\bar{N}$, as a function of the amplifier input power $P_{I N}$

a $N_{0}$

$c N_{L}$

$P_{I N}$. The calculations have been done starting from the corresponding exprs. $10 a, 10 b$ and 6 , making use of the experimental data given in Section 3. One can see how $N_{0}$ and $N_{L}$ diverge when $P_{I N}$ increases. It must be pointed out that the power measured at the amplifier output was optically filtered to $0.1 \mathrm{~nm}$ by the optical spectrum analyser we used, and hence the measured output power can be considered as a signal power. For this reason, a fraction of the spontaneous emission coupled to the amplified signal has been included in the value of $\boldsymbol{P}_{\text {oUT }}$ in eqn. $10 \mathrm{~b}$.

In fact, the obtained value of $\bar{N}$ corresponds to a certain carrier density in some internal co-ordinate of the amplifier. Another a priori chosen expansion of $R(N)$ would give results corresponding to a more or less similar co-ordinate.

Starting from eqns. 13 and 15 , both the saturation parameter $P_{s}$ (Fig. 3) and the effective carrier lifetime $\tau_{c}$ (Fig. 4) have been calculated. According to these results, one can observe that both parameters tend to a constant value when the input power decreases. Note that the 
values of $P_{s}$ and $\tau_{c}$ corresponding to $P_{I N}=-26 \mathrm{dBm}$ are close to the theoretical values (denoted $P_{s}^{o}$ and $\tau_{c}^{o}$ in Figs. 3 and 4 , respectively) corresponding to a small signal regime (linear behaviour of the TWOA)

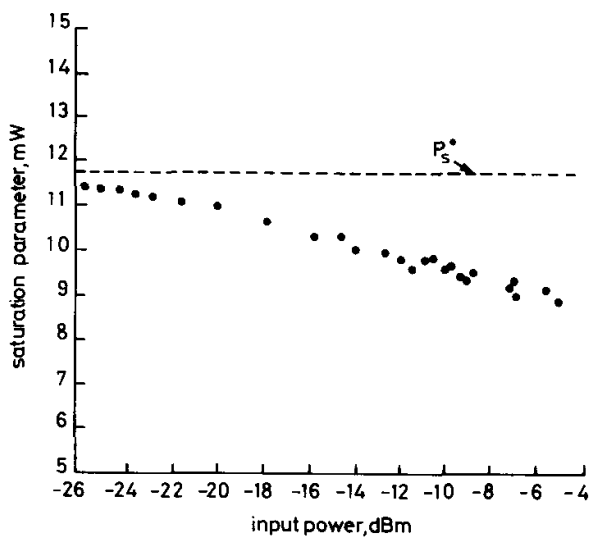

Fig. 3 Saturation parameter $P_{s}$ as a function of the amplifier input optical power $P_{I N}$, calculated from eqn. 13

All parameters are listed in Table 1

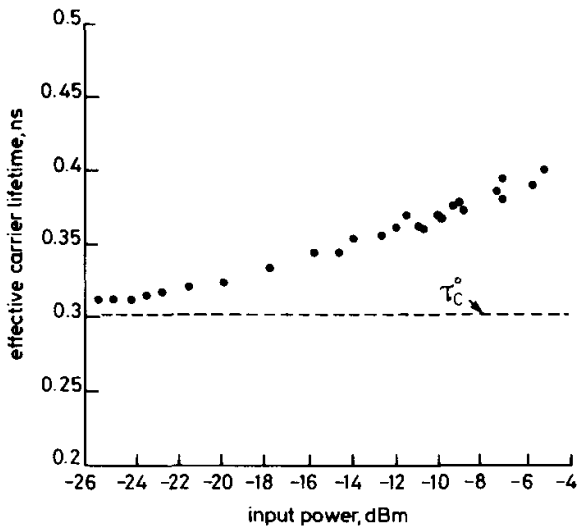

Fig. 4 Effective carrier lifetime $\tau_{c}$ as a function of the amplifier input optical power $P_{I N}$, calculated from eqn. 15

All parameters are listed in Table 1

On the other hand, if the incident optical power increases in an appreciable way (in our experiment we reached $-5 \mathrm{dBm}$ ), there are important variations of both parameters in relation to their small signal values $\left(P_{s}^{o}=\right.$ $11.75 \mathrm{~mW}$ and $\tau_{c}^{\circ}=0.3 \mathrm{~ns}$ ). Related to the saturation parameter, there is a progressive decay of about $125 \mu \mathrm{W} /$ $\mathrm{dBm}$. Logically, the effective carrier lifetime suffers a corresponding increase (about $4.5 \mathrm{ps} / \mathrm{dBm}$ for this amplifier). Note that these slopes are only approximated and, obviously, they do not take into account the fluctuations observed in Figs. 3 and 4. It has already been mentioned in the preceding Section that these fluctuations are due to the behaviour of the laser source we used, which showed some instabilities at high power levels.

We wish to emphasise that we have also realised numerical simulations of the power propagation equation for the TWOA (using the values $\boldsymbol{P}_{s}$ obtained for each value of $\left.\boldsymbol{P}_{I N}\right)$. The theoretical $\boldsymbol{P}_{I N}-\boldsymbol{P}_{\text {ovT }}$ characteristic calculated is shown in Fig. 5 and it is compared to the experimental one. We can observe that the theoretical results are in good agreement with those experimentally measured, although, from a rigorous point of view, both curves present few differences. Perhaps this is owing to mechanisms not considered here, such as, for example, the intervalence band absorption, or else, to small errors in the measurements we have done.

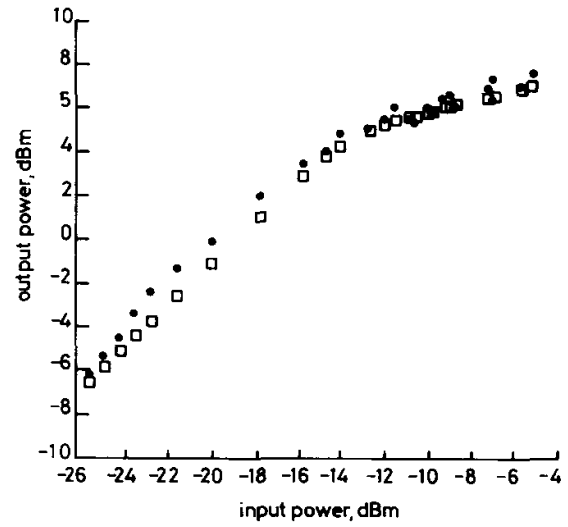

Fig. 5 Theoretical and experimental $P_{I N}-P_{\text {ovT }}$ characteristics $\square$ Theoretical

- Experimental

\section{Conclusions}

In this paper, a practical method has been developed to estimate the values of the saturation parameter and the effective carrier lifetime starting from experimental measurements. The results show that, actually, both magnitudes are not intrinsic parameters of the optical amplifier characterised as they depend on the injected optical power. Moreover, because $R(N)$ must be linearly approximated to establish a definition of $P_{s}$, the value of this parameter will depend on the expansion of $R(N)$ under consideration. It is only possible to establish an absolute formula if one assumes (a priori) that $\tau_{c}$ is a constant. For example, considering the total carrier recombination, the results obtained show that $P_{s}$ can vary around $0.125 \mathrm{~mW} / \mathrm{dBm}$ (more or less) when $P_{I N}$ is greater than $-20 \mathrm{dBm}(10 \mu \mathrm{W})$. This could be very important in WDM systems or booster structures incorporating semiconductor optical amplifiers. Finally, we think that the method exposed here proves to be useful as it gives a set of simple analytical expressions (the most complicated calculation is the solution of a third-order polinomial in eqns. 10).

\section{References}

1 ADAMS, M.J., COLLINS, J.V., and HENNING, I.D.: 'Analysis of semiconductor laser optical amplifiers', IEE Proc. $J, 1985,132$, pp 58-63

2 SIMON, J.C.: 'GaInAsP semiconductor laser amplifiers for singlemode fiber communications', IEEE J. Lightwave Technol., 1987, LT-5, pp. 1286-1295

3 O'MAHONY, M.J: 'Semiconductor laser optical amplifiers for use in future fiber systems', IEEE J. Lightwave Technol., 1988, LT-6, pp. $531-544$

4 EINSENSTEIN, G., TESSLER, N., KOREN, U., WIESENFELD, J.M., RAYBON, G., and BURRUS, C.A.: 'Length dependence of the saturation characteristic in $1.5-\mu \mathrm{m}$ multiple quantum well optical saturation characteristic in 1.5- $\mu$ m multiple quantum well optic

5 amplifiers', IEEE Photonics Technol. Lett., 1990, 2, pp. 790-791 5 KOMORI, K., ARAI, S., and SUEMATSU, Y.: 'Noise in semicon-
ductor laser amplifiers with quantum box structure', IEEE Photonics Technol. Lett., 1991, 3, pp. 39-41

IEE PROCEEDINGS-J, Vol. 140, No. 1, FEBRU ARY 1993 
6 RUIZ-MORENO, S., GUITART, J., and SONEIRA, M.J. : 'Modelling and characterisation of a nonlinear travelling wave optical amplifier'. Deliverable 143, RACE Project 1027 WP.4 (Optical Amplifiers), 1991

7 JOPSON, R.M., and DARCIE, T.E.: Chap. 8, in YAMAMOTO, Y (Ed.): "Coherence, amplification, and quantum effects in semiconductor lasers' (Wiley-Interscience, 1991)

8 STORKFELT, N MIKKELSEN, B OLESEN, DS YAMAGUCHI, M., and STUBKJAER, K.E.: 'Measurement of carrier life- time and linewidth enhancement factor for $1.5-\mu \mathrm{m}$ ridge-waveguide laser amplifier', IEEE Photonics Technol. Lett., 1991, 3, pp. 632-634 9 GUITART, J., RUIZ-MORENO, S., and JUAN, L.: 'Theoretical and experimental characterization of the non-linear behaviour of a travelling wave semiconductor optical amplifier'. Presented at SIOF 92, Cardiff, 3th March-1st April 1992

10 FERNIER, B., BROSSON, P. JICQUEL, J.-P., and BENOIT, J.

'15 $\mu \mathrm{m}$ laser with high external quantum efficiency and controlled emission wavelength', IEE Proc. J, 1987, 134, pp. 27-34 